\title{
New Molecular Targets in Metastatic Prostate Cancer
}

Francesco Pantano1,2, Giulia Ribelli2, Michele Iuliani ${ }^{2 *}$, Marco Fioramonti², Mark Leakos², Alice Zoccoli², Daniele Santini, ${ }^{1,2}$, Giovanni Muto3, Giuseppe Tonini',2

\footnotetext{
${ }^{1}$ Medical Oncology, Campus Bio-Medico University of Rome, Rome, Italy

${ }^{2}$ Translational Oncology Laboratory, Campus Bio-Medico University of Rome, Rome, Italy

${ }^{3}$ Department of Urology, Campus Bio-Medico University of Rome, Rome, Italy

Email: *m.iuliani@unicampus.it
}

Received 13 March 2016; accepted 14 June 2016; published 17 June 2016

Copyright (C) 2016 by authors and Scientific Research Publishing Inc.

This work is licensed under the Creative Commons Attribution International License (CC BY).

http://creativecommons.org/licenses/by/4.0/

c) (i) Open Access

\begin{abstract}
Prostate cancer (PCa) is the second leading cause of cancer death in men. Despite initial responses, almost all patients progress to castration-resistant prostate cancer (CRPC). Over the past decade, increased understanding of the mechanisms that drive resistance to castration has led to the development of next-generation androgen receptor targeting agents such as abiraterone acetate and enzalutamide. Moreover in the last few years, results from large Phase III trials led to the approval of an $\alpha$-emitter (radium-223), the bone resorption-targeting drug denosumab and an immunotherapy (sipuleucel-T) that showed improvements in terms of overall survival. In the field of metastatic CRPC, other novel therapeutics have recently been proven to extend survival via distinct mechanisms of action such as the new and more potent classes of androgen inhibitors, ortonel, ARN-509 and galeterone, the endothelin A receptor antagonist zibotentan, the Src inhibitor dasatinib, the c-MET inhibitor cabozantinib and the immune checkpoint inhibitor ipilimumab. This review aims to revisit the evolution of androgen receptor targeting therapeutics and to discuss other important alternative biologic pathways that have given rise to new agents in metastatic prostate cancer.
\end{abstract}

\section{Keywords}

Prostate Cancer, Target Therapy

\footnotetext{
${ }^{*}$ Corresponding author.
}

How to cite this paper: Pantano, F., Ribelli, G., Iuliani, M., Fioramonti, M., Leakos, M., Zoccoli, A., Santini, D., Muto, G. and Tonini, G. (2016) New Molecular Targets in Metastatic Prostate Cancer. Journal of Cancer Therapy, 7, 388-401. 


\section{Introduction}

Prostate cancer (PCa) is currently the second leading cause of cancer-related deaths in men in the United States and the most common cancer in elderly males in Europe [1] [2]. The main predictors of prostate cancer risk are age, race or ethnicity, and family history. There are numerous molecular, genetic, environmental, and dietary factors, with varying degrees of supporting evidence. Although prostate cancer typically manifests in old age, a growing body of evidence suggests that prostatic carcinogenesis is initiated much earlier. Prostatic intraepithelial neoplasia (PIN) is the histologic entity widely considered to be the most likely precursor of invasive prostate cancer. Although not all patients with high-grade PIN (HGPIN) progress to develop invasive disease, PIN is characterized by cellular proliferation within pre-existing ducts and glands, with cytologic changes that mimic those of cancer [3]. PIN is associated with progressive abnormalities of phenotype and genotype that are intermediate between normal prostatic epithelium and cancer [3]. The majority of men diagnosed with prostate cancer will benefit from not being treated, because they have low-volume indolent tumors that do not require immediate treatment. Active surveillance is a reasonable and widely accepted approach for these patients [4]. On the other hand localized PCa is the category of men presenting either with a high-risk localized cancer or with metastatic disease that are usually treated aggressively with prostatectomy, radiation therapy and/or androgen deprivation therapies (ADT). ADT is the standard of care for advanced prostate cancer. This treatment drives to prostate-specific antigen (PSA) decrease and clinical improvements in more than $90 \%$ of patients [5]. Nevertheless, this therapeutic approach is not curative and the majority of patients often develop castration resistance. Moreover, most men with advanced prostate cancer are at high risk for developing bone metastases [6]. Bone metastases are associated with a reduced quality of life and an increased risk of complications such as pathological fractures, spinal cord compression, radiation or surgery to the bone that are collectively defined as skeletalrelated events (SREs) [7].

In the last years, the introduction of highly effective novel treatments has significantly changed the management of metastatic castration resistant prostate cancer (mCRPC) patients with an improved overall survival (OS) advantage [8]. These agents include a chemotherapeutic (cabazitaxel), two hormonal agents (abiraterone and enzalutamide), an alpha-emitting bone-seeking radioisotope (radium-223) and an immunotherapeutic agent (sipuleucel-T). In particular in 2011, the novel tubulin-binding taxane, cabazitaxel, as a second-line chemotherapy showed encouraging results [9]. Between 2011 and 2012, abiraterone acetate and enzalutamide showed further OS improvements [10] [11] as well as in 2013, radium-223 became available in clinical practice [12]. In 2011 it was demonstrated that treatment with denosumab significantly prolonged the median time to the first SRE compared to zoledronic acid in CRPC patients suffering with bone metastases [13]. Finally, immunotherapy with sipuleucel-T, an immune-modulating agent (FDA approved in 2010) showed a survival advantage [14], but it was not widely used because, rarely, patients responded to therapy.

Advances in the understanding of the biology of metastatic PCa have led to major improvements in the area of targeted therapies, especially in recent years. Indeed, novel agents now in clinical evaluation include AR inhibitors (ARN509, ODM-201), new generation hormonal therapies (galeterone, orteronel) immunotherapies (like ipilimumab) and drugs targeting key molecular pathways involved in tumor progression and bone metastases onset (cabozantinib, dasatinib, saracatinib, zibotentan).

This review provides an overview of the current therapeutic options for the treatment of mCRPC, including novel agents currently in clinical evaluation.

\section{Targeting the Androgen Pathway}

Recent evidence has demonstrated that androgen-based pathways remain a key signal in the progression of CRPC. Indeed, tumor tissues express several enzymes that regulate the synthesis of testosterone and dihydrotestosterone, such as cytocrome P450 17 alpha hydroxysteroid dehydrogenase (CYP17) [15]. Persistent androgen signaling represents an effective therapeutic target in mCRPC.

Several preclinical and clinical data confirmed that the shift from endocrine-dependent to intracrine androgen signaling progression is essential for the progression of prostate cancer and for the resistance to main androgen deprivation treatments [16] [17]. Furthermore during mCRPC progression, androgen receptor (AR) undergoes molecular changes, such as overexpression, mutation, alternative splicing, post-translational modifications and interactions with other pathways (non-classical AR signaling) [18] [19]. 


\subsection{Abiraterone}

Abiraterone acetate is an orally administered steroidal antiandrogen derived from the structure of pregnenolone. It inhibits androgen biosynthesis blocking the hydroxylase and lyase activities of CYP17A with 10 - 30 fold stronger than ketoconazole [20]. Consequently, serum and intratumoral androgen production in the adrenal gland, testes and prostate cancer cells became undetectable [21] [22]. Abiraterone is usually administered with prednisone to ameliorate the secondary increase of the adrenocorticotropic hormone that can lead to excess mineralocorticoid synthesis [23].

Two randomized Phase III trials demonstrated that abiraterone improved OS compared with placebo. In the COU-AA-301 trial [10], 1195 patients previously treated with docetaxel were randomized to abiraterone plus prednisone or placebo plus prednisone. The primary endpoint of OS was met with an improvement in OS of 3.9 months compared with placebo. In the COU-AA-302 trial, 1088 chemotherapy-naïve patients were randomized to abiraterone plus prednisone or to placebo plus prednisone. The results showed an 8.2-month improvement in radiographic progression free survival (PFS) favoring abiraterone. Abiraterone treatment was also associated with better pain control from skeletal metastases, a delay in development of SREs, and delayed radiological skeletal progression. More specifically, $25 \%$ of patients developed a skeletal event in 9.9 months when treated with abiraterone versus 4.9 months with placebo, and the time to first SRE was 25.0 months with abiraterone compared to 20.3 months with placebo [24] [25]. Abiraterone was well tolerated in this trial although adverse effects due to increased mineralocorticoid levels secondary to CYP17 blockade were more common with abiraterone. Specific effects that were significantly higher with abiraterone than placebo included fluid retention and edema (31\% vs $22 \%$ ) and hypokalemia ( $17 \%$ vs $8 \%$ ) [25]. These abiraterone benefits on metastatic bone disease may be attributable not only to a direct antitumor effect, but also to specific action on the bone microenvironment. Indeed, recently a direct anabolic and an anti-reabsorptive effect on bone by abiraterone, both in vitro and in mCRPC patients was found. Our research team demonstrated that abiraterone was able to specifically modulate osteoclasts and osteoblasts leading to direct anabolic and anti-reabsorptive effects both in presence and absence of steroids, suggesting a non-canonical mechanism of action that seems to be, at least in part, androgenindependent [26].

\subsection{Enzalutamide}

Enzalutamide is another promising oral AR inhibitor that targets multiple steps in the AR signaling pathway. Two Phase III trials have demonstrated the efficacy of enzalutamide in the treatment of patients with mCRPC.

A randomized Phase III trial (AFFIRM) showed that mCRPC patients treated with enzalutamide after docetaxel had a significant improvements in OS compared to placebo group. Further benefits concerned the delay in time to first SRE and improvements in bone pain and quality of life [11].

Moreover, in the Phase III PREVAIL study evaluating enzalutamide versus placebo in patients with mCRPC, who had not received chemotherapy, the anti-androgen significantly reduced the risk of bone disease progression and death. The study also showed significant improvements in other secondary and prespecified exploratory end points, such as delayed start of chemotherapy, lower risk of first SRE and an increase of the percentage of responded compared with placebo [27]. Moreover enzalutamide is reported to cause fatigue (11\%), hot flashes (20\%), headache (12\%), nausea, diarrhea, constipation and musculoskeletal pain [11].

Ongoing trials could further define the optimal use of abiraterone acetate in combination with enzalutamide for treating mCRPC patients.

\subsection{Orteronel}

Orteronel (TAK-700), like abiraterone, is an androgen production inhibitor with a selectivity for 17 - 20 lyase over 17- $\alpha$-hydroxylase, but with fewer mineralcorticoid effects. Moreover, for long-term therapy orteronel could be administrated with prednisone [27].

A Phase I/II open-label study in patients with mCRPC showed that orteronel treatment decreased PSA, testosterone and dehydroepiandrosterone levels [28]. The majority of side effects were lower grade even if some high-grade events were reported such as hypertension (in 13\% of patients), shortness of breath (in $8 \%$ ), and pneumonitis (in 5\%) [28].

Currently, two ongoing randomized, placebo-controlled Phase III trials are assessing orteronel in patients with 
progressive CRPC who are either chemotherapy naive or pretreated with docetaxel. Unfortunately no improvement in OS was observed in treated patients compared with placebo, although the treatment led to longer relative PFS [29].

\subsection{Galeterone}

Galeterone (TOK-001) is an androgen biosynthesis inhibitor with a biological effect that changes with its concentration: at low concentrations, it blocks CYP17A1 activity; at moderate concentrations, it acts as an AR antagonist; and at high concentrations, it drives to AR degradation [26]. Based on phase I trial (ARMOR1) data, galeterone received fast-track designation from the FDA for the treatment of mCRPC [30]. Most side effects were minor and included fatigue, nausea and diarrhea, but nothing requiring cortisol treatment [30]. The Phase II ARMOR2 trial in 25 progressive CRPC patients confirmed safety and efficacy and showed a 4.8-month median OS benefit compared with placebo [31].

\subsection{ARN-509}

ARN-509 is a second generation AR inhibitor that binds to AR preventing growth and androgen-mediated gene transcription in vitro, through the inhibition of AR nuclear translocation and its consequent DNA bond [32]. Phase I clinical trials demonstrated that ARN-509 decreased PSA levels of $\geq 50 \%$ from baseline at 12 weeks in $46.7 \%$ of treated patients. The toxicity profile included fatigue (38\%), nausea (29\%) and pain (24\%). It is currently being evaluated in Phase II clinical trials and published data shows a significant PSA response [33].

\subsection{ODM-201}

ODM-201 is another promising prostate cancer treatment that, with its major metabolite, ORM 15341, specifically inhibits AR nuclear translocation [34]. ODM-201 showed a higher anticancer activity compared with enzalutamide in xenograft models of prostate cancer.

In a Phase II randomized trial, 124 treated patients displayed a clinical benefit in terms of overall response rate and bone response [35]. The most common treatment side events were fatigue or asthenia (12\%), hot flush (5\%), and decreased appetite (4\%).

\section{Targeting Bone Microenvironment}

Novel therapies in the management of prostate cancer target both the cancer cells as well as the bone microenvironment, preferential site of prostate cancer metastases.

Prostate bone metastases are typically osteoblastic, thus characterized by both an osteoblastic proliferation with enhanced matrix deposition and an increased osteoclastic activity [36] [37]. The result is an increase of osteoblast proliferation and differentiation which increase the deposition of abnormal woven bone [38]. Enhanced osteolytic activity causes the release of growth factors stored in the bone matrix into the tumor microenvironment that stimulate tumor cell growth and alter their phenotype, thus promoting a vicious cycle of metastasis and bone pathology. Increased osteolysis makes SREs a very common feature of bone metastatic prostate cancer patients. SREs are very uncommon when the disease is androgen sensitive, nevertheless their incidence increase when prostate became castration resistant [39].

In this regard, bisphosphonates and denosumab currently represent two effective additional approach in the management of metastatic prostate cancer and several randomized, controlled trials supported their efficacy in reducing skeletal morbidity of patients [40].

\subsection{Bisphosphonates}

There are two groups of bisphosphonates, non-nitrogen-containing and nitrogen-containing, with different effects on osteoclasts. Etidronate, clodronate and tiludronate belong to the non-nitrogen-containing class of bisphosphonates, whereas pamidronate, alendronate, ibandronate, risedronate and zoledronic acid belong to the nitrogen-containing bisphosphonates (which are more potent osteoclast inhibitors and more commonly used). Bisphosphonates drive to osteoclast apoptosis, affecting their differentiation and maturation and thus inhibiting their bone resorption activity. Moreover bisphosphonates influence macrophages, gamma delta T cells, osteob- 
lasts and tumor cells as demonstrated in preclinical models. In addition to their effects on host cells, bisphosphonates could also have antitumor and/or antiangiogenic effects [41].

Zoledronic acid is currently approved for the treatment of patients with bone metastatic prostate cancer that is progressing while on initial hormone therapy. A phase III trial supported the efficacy of zoledronic acid in this subset of patients that after the treatment showed a significant decrease of SREs incidence, a longer median time to develop SREs and better pain scores [42].

Osteoclastic proliferation, differentiation, activation and apoptosis is regulated by RANK (Receptor Activator of Nuclear Factor-kB), RANKL (Receptor Activator of Nuclear Factor-kB Ligand) and the decoy Receptor Osteoprotegerin (OPG). These factors belong to TNF and TNF receptor superfamily and have a central role in the establishment of bone metastases. Indeed RANKL induce osteoclast-mediated bone resorption and the consequent release of matrix growth factors such as Tumor Growth Factor-b (TGF-b) and platelet-derived growth factor (PDGF) that in turn enhance the growth of tumor cells establishing a positive feedback mechanism [43].

Finally, it has been demonstrated that RANK is expressed not only on osteoclasts, but also on prostate cancer cells [44] suggesting that RANK allows cancer cells to migrate where RANKL is abundantly expressed, like the bone.

\subsection{Denosumab}

Denosumab (AMG162) is a human non-cytotoxic IgG2 monoclonal antibody with an extremely high affinity and specificity for human RANKL. It is approved for the treatment of osteoporosis, cancer treatment induced bone loss, bone metastases and other skeletal pathologies mediated by osteoclasts.

In a castration-resistant prostate cancer population presenting with bone metastases, the median time-to-first SRE for the denosumab arm was significantly prolonged (21 months) compared to the zoledronic acid arm (17 months) with no improvements in OS or progression of disease [13].

Moreover, in a Phase III trial in men with nonmetastatic castration-resistant prostate cancer with a high risk of developing bone metastases, denosumab significantly increased bone-metastasis-free survival by a median of 4 months compared to placebo ( 29 vs 25 months) potentially confirming the role of RANK/RANKL in regulating cancer cell homing to the bone [13].

\subsection{Safety of Bone Target Therapies}

One of the most commonly reported adverse event related to bisphosphonates and denosumab treatment is hypocalcaemia that is most often asymptomatic with these agents. In particular, hypocalcaemia occurred more frequently with denosumab than with zoledronic acid as shown in the Phase III trial in patients with CRPC and bone metastases (13\% vs 6\%) [13]. In an integrated analysis of 5723 patients from three randomized Phase III trials, the safety profile for denosumab was better than for zoledronic acid, demonstrating no effect on renal function and no need for dose adjustment or renal monitoring [45]. In patients receiving zoledronic acid the incidence of hypocalcaemia was lower than in patients receiving denosumab (1.3\% vs $3.1 \%$ for grade 3 or grade 4 toxicities), though most cases were asymptomatic [45]. Thus, repletion of vitamin $\mathrm{D}$ levels before and during the therapy and monitoring of calcium levels during therapy is recommended in the prescribing information of denosumab.

\subsection{Radiopharmaceuticals}

Radiopharmaceuticals are other interesting agents targeting bone metastases; several studies showed how betaemitting radiopharmaceuticals allowed bone pain relief in mCRPC patients due to their similarity to calcium, emitting radiation when they were taken up at site of osteoblastic activity.

Stronzium-89 and samarium-153 were the first radiopharmaceuticals approved for bone metastases pain relief in patients with mCRPC [46]. Although these radiopharmaceuticals are useful tool for pain palliations, no study showed impact on OS. One randomized control trial showed that stronzium-89, after six cycles of docetaxel, improved clinical PFS but frequent hematological adverse events [47] limits their use only to symptomatic patients with multiple bone metastases.

Radium-223 is an alpha emitter that differs from beta emitter agents since it delivers a highly localized radiation to the bone surface, causing double-stranded DNA breaks that lead to cell death giving less irradiation to 
healthy bone marrow than beta-emitters [48]. It is a calcium mimetic molecule that forms a complex with hydroxyapatite, which forms $50 \%$ of bone matrix; this linking allows radium 223 to be incorporated into the bone matrix emitting alpha particle preserving the health of bone tissue and bone marrow and limiting distribution to soft tissue [49].

Radium-223 was recently approved by FDA for men with symptomatic mCRPC with only bone metastases showing a significant impact on OS in patients who progress with docetaxel or unfit to docetaxel. Several Phase I and II trials showed safety and tolerability of alpharadin, radium-223 chloride in solution, in mCRPC patients, with improvements in bone turnover markers such as bone alkaline phosphatase (bALP) and urine N-telopeptide (uNTX) [50] [51]. These results led investigators to conduct a randomized open-label, multicenter Phase III trial evaluating the impact on OS of radium-223 in mCRPC patients with bone metastases previously treated with docetaxel or unfit to receive docetaxel. This Phase III trial was stopped early after pre-planned efficacy interim analysis, since OS was significantly improved in the radium-223 arms versus placebo-control arm (median, 14.0 vs 11.2 months respectively); updated analyses in all 921 patients, performed before crossover from placebo to radium-223, showed a similar survival advantage for radium-223 treatment (median, 14.9 vs 11.3 months) [12]. Moreover, radium-223 showed efficacy in all secondary end points including time to the first symptomatic skeletal events (median, 15.6 months vs 9.8 months, respectively). The side effects of radium 223 can include diarrhoea and sickness but these are generally mild. Starting from these promising results, new trials are under investigation to better understand combination therapy with docetaxel and other new emergent therapies such as abiraterone acetate that will improve OS in this subset of patients.

\section{Targeting Signal Transduction Pathways}

\subsection{Targeting c-MET/HGF Pathway}

The receptor tyrosine kinase MET and its ligand hepatocyte growth factor (HGF) signaling pathway promotes stemness phenotype, tumor growth, invasion and metastases in several malignancies.

MET is expressed by primary and metastatic prostate carcinomas and its levels are higher in bone metastases compared with lymph node metastases or primary tumors [52]. Also osteoblasts and osteoclasts express MET and HGF regulating cellular responses, such as proliferation, migration and differentiation.

Cabozantinib (XL184) is an orally tyrosine kinase inhibitor that targets MET and VEGF receptor 2 (VEGFR2). In a multicenter, Phase II, nonrandomized expansion study of men with CRPC, bone metastases and disease progression despite docetaxel treatment, Cabozantinib was associated with improvements in bone scans, patient reported pain and analgesic use, circulating tumor cells, and bone biomarkers. The study was stopped because of these improvements in bone response. Patients treated with Cabozantinib showed a significant improvement in the primary end point of PFS compared with placebo group (median, 23.9 vs 5.9 weeks, respectively) [53]. Instead, in the following Phase III trial (COMET-1), Cabozantinib did not demonstrate a statistically significant increase in OS compared to prednisone. Indeed, COMET-1 showed a median OS of 11 months for treated patients and 9.8 months for the prednisone arm [54]. The most commonly reported adverse events with Cabozantinib included fatigue (13\%), diarrhea (5\%), and hypertension (5\%) [54].

\subsection{Targeting Cellular Src Kinase}

The membrane-associated tyrosine kinase Src (encoded by the c-Src gene) is a proto-oncogene involved in the onset of several pathological processes such as tumor cell proliferation, adhesion, invasion, migration and metastasis development [55] [56].

One of the most studied Src inhibitor is dasatinib. In in vivo model of prostate cancer, it showed a synergistic effect when administered with docetaxel inhibiting the proliferation of prostate cancer cells implanted into bone [57].

A Phase II study of dasatinib in combination with docetaxel demonstrated safety and activity becoming the subject of the Phase III READY trial [58]. This study enrolled 1500 men with chemotherapy-naive mCRPC and randomized them to docetaxel and prednisone with or without dasatinib $100 \mathrm{mg}$ daily. In this trial, mCRPC men naïve for chemotherapy and treated with dasatinib plus docetaxel did not show OS improvement. Indeed, the median OS in dasatinib and placebo group was 21.5 and 21.2 months respectively [59]. The most common grade 3 - 4 adverse events included diarrhea (8\%) patients in the dasatinib group vs $4 \%$ patients in the placebo group, 
fatigue (8\% vs $6 \%$ ), and asthenia (5\% vs 3\%) [59].

\subsection{Targeting Endothelins}

Endothelins (ET-1, ET-2, ET-3) are a group of 21-amino acid peptides that are produced in a variety of tissues, where they regulates the vasomotor tone, nociception, hormone production and cell proliferation [60]. It has been demonstrated that circulating levels of endothelin-1 (ET-1) in metastatic prostate cancer patients increased compared to patients with localized disease [60]. In the bone microenvironment, ET-1 alters osteoblasts/osteoclasts balance driving to new bone deposition that is typical of prostate cancer metastases [61]. Indeed, malignant cells release ET-1 that binds its receptor (Endothelin receptor A), expressed by osteoblasts, stimulating their proliferation and bone apposition activity. Activated osteoblasts in turn release several growth factors promoting survival and growth of bone metastatic cancer cells.

Zibotentan (ZD4054) is an oral, specific Endothelin receptor A antagonist under investigation in ENTHUSE clinical trials. In ENTHUSE M1 study in men with mildly symptomatic CRPC zibotentan treatment compared to placebo did not significant improve OS (24.5 vs 22.5 months, respectively) [62]. Moreover, the ENTHUSE M0 study evaluating zibotentan treatment in patients with non-metastatic CRPC has been discontinued because did not meet its primary end points (OS and progression-free survival [PFS]).

Finally, randomized Phase III ENTHUSE M1C trial investigating the effect of zibotentan in combination with docetaxel versus docetaxel plus placebo showed no improvements in OS, PSA response rate, time to PSA progression, PFS, time to new bone metastases, time to new SREs, pain response, or time to pain progression [63]. The most commonly reported adverse events in zibotentan-treated patients were peripheral edema (37.7\%), headache (26.2\%) and nasal congestion (24.9\%); each occurred with $>15 \%$ higher incidence than in the placebo group [63]. In view of these results, no further investigations with zibotetan are ongoing.

\subsection{Immunotherapies}

Prostate cancer represents an appealing setting for immunotherapy approaches given the relatively high expression of several tumor-associated antigens (TAAs) [64]. In this regard Sipuleucel-T has recently been approved in mCRPC and alternative strategies based on the possibility of interfering with the phenomenon of tumor immune escape are currently in development phases. The most promising approach appears to be the modulation of immunosuppressive micro-environment by acting on their co-inhibitory molecule [65]. Nowadays, CTLA-4 (cytotoxic T-lymphocyte associated antigen 4) is currently the most investigated, and the pharmacological approaches aimed at its inhibition are in advanced stages of clinical investigation also in metastatic prostate cancer [66] [67].

\subsection{Sipuleucel-T}

Sipuleucel-T is an autologous cellular immunotherapy indicated for the treatment of asymptomatic or minimally symptomatic metastatic CRPC. The patient's peripheral blood mononuclear cells are treated with a prostatic acid phosphatase-granulocyte macrophage colony-stimulating factor (PAP-GM-CSF) fusion protein in addition to various other cytokines to generate PAP-specific T cells capable of recognizing and killing prostate cancer cells that express PAP. This treatment was FDA-approved in 2010 for use in patients in mCRPC, based on the results of the pivotal Phase III trial (IMPACT). In this study 512 patients with asymptomatic or minimally symptomatic chemotherapy-naive mCRPC were randomized to receive sipuleucel-T versus placebo in a 2:1 ratio showing 4.1-month improvement in median OS (25.8 vs. 21.7 months) for sipuleucel-T compared with control (HR = 0.78; $95 \%$ CI: $0.61,0.98$ ) [68]. Common adverse reactions reported during a safety evaluation of 601 patients who received sipuleucel-T were chills, fatigue, fever, back pain, nausea, joint ache, and headache. The majority of adverse reactions were mild or moderate in severity [68].

\subsection{Ipilimumab}

Ipilimumab is a human monoclonal antibody that enhances and prolongs T-cell activation by blocking immune checkpoint CTLA-4 receptors found on the surface of T cells [65]. In a randomized Phase II trial, 108 patients with advanced prostate cancer treated with ipilimumab plus androgen-deprivation therapy showed undetectable PSA levels by 3 months compared with patients treated with endocrine therapy alone (55\% vs 38\%) [66]. 
Recently, the results from a randomized, double-blind Phase III study (CA-184-043) comparing ipilimumab with placebo following bone-directed radiation therapy in CRPC patients previously treated with docetaxel demonstrated no improvement in OS [67]. The most frequent grade 3-4 adverse events included diarrhea (16\% in the ipilimumab group vs $2 \%$ in the placebo group), fatigue ( $11 \%$ vs $9 \%$, anemia ( $10 \%$ vs $11 \%$ ), and colitis ( $5 \%$ vs 0 ) [67].

Nevertheless, a subgroup analysis suggests that ipilimumab may be most active in patients with favorable laboratory prognostic factors (e.g., decreased alkaline phosphatase or elevated hemoglobin level) or in patients without visceral disease [66] (Table 1).

Table 1. Metastatic prostate cancer therapies currently approved or in clinical development.

Compound Company $\quad$ Structure $\quad$ Stage of development

\section{Targeting the androgen pathway}

$\begin{array}{cc}\text { Abiraterone } & \text { Janssen } \\ \text { Enzalutamide } & \text { Astellas } \\ \text { Orteronel } & \begin{array}{c}\text { Takeda Pharmaceutical } \\ \text { Company }\end{array} \\ \text { Galeterone } & \text { Tokai Pharmaceuticals } \\ \text { ARN-509 } & \text { Johnson \& Johnson } \\ \text { ODM-201 } & \begin{array}{c}\text { Orion and Bayer } \\ \text { Health Care }\end{array}\end{array}$

Targeting bone microenvironment

$\begin{array}{ll}\text { Zoledronic Acid } & \text { Novartis } \\ \text { Denosumab } & \text { Amgen } \\ \text { Radium-223 } & \text { Bayer }\end{array}$

\section{Targeting signal transduction pathways}

$\begin{array}{cc}\text { Cabozantinib } & \text { Exelixis } \\ \text { Dasatinib } & \text { Bristol-Myers Squibb } \\ \text { Zibotentan } & \text { AstraZeneca }\end{array}$

$$
\begin{aligned}
& \text { Oral small molecule TKi } \\
& \text { Oral small molecule TKi } \\
& \text { Oral small molecule }
\end{aligned}
$$

Immunotherapies

$\begin{array}{ll}\text { Sipuleucel-T } & \text { Dendreon Corporation } \\ \text { Ipilimumab } & \text { Bristol-Myers Squibb }\end{array}$

Intravenous autologous cellular immunotherapy

Intravenous monoclonal antibody

Other molecules

Tasquinimod

$$
\begin{gathered}
\text { Ipsen and Active } \\
\text { Biotech }
\end{gathered}
$$

Custirsen

Oncogenex Pharma
Oral small molecule tumor microenvironment inhibitor

Intravenous antiapoptotic signalling inhibitor
Phase III

Phase III

Phase III

Phase III

Phase III

Phase III

Phase IV

Phase IV

Phase III

Phase III

Phase III

Phase III

Phase III

Phase III

Phase III

Phase III
17 a-hydroxylase/C17,20-lyase (CYP17) inhibitor

Androgen receptor antagonist

CYP17A1 inhibitor

Both androgen receptor antagonist and an CYP17A1 inhibitor

Androgen receptor antagonist

Androgen receptor antagonist

Farnesyl pyrophosphate sy50*ntetase inhibitor

RANK-L antibody

Radiopharmaceutical alpha-particles target 


\section{Other Molecules}

\subsection{Tasquinimod}

Tasquinimod is a novel immunotherapy, orally active quinoline-3-caboxamide analog that targets the tumor microenvironment exerting immunomodulatory and antiangiogenic properties [69]. In particular, tasquinimod interferes with vascular tissue homeostasis downregulating the angiogenic suppressor thrombospondin-1 and upregulating Hypoxia-inducible factor 1-alpha (HIF-1 $\alpha$ ). At the same times it is an inhibitor of S100A9 (a protein from the family of calcium-binding S100 proteins expressed on myeloid-derived suppressor cells) modulating the local tumor immunity. Indeed, it prevents the bind of S100A9 protein with its ligand inactivating proinflammatory cascade signaling pathways [70].

In a randomized phase II trial chemotherapy-naïve men with castrate-resistant prostate cancer showed a significant increase in progression free survival and overall survival with tasquinimod compared with placebo [71]. Tasquinimod was considered safe, with low to moderate side effects, which included mild gastrointestinal issues, muscle and joint pains, and fatigue [71]. Anyway, a phase III trial in 1245 patients did not confirm survival advantage. Even if there was a significant improvement in progression-free survival (median, 7.0 versus 4.4 months), there was no benefit in overall survival (median, 21.3 versus 24.0 months, 95\%) [72].

\subsection{Custirsen}

Custirsen (OGX-011) is a second-generation, 2'-methoxyethyl-modified phosphorothioate antisense oligonucleotide that inhibits clusterin expression [73]. Clusterin is an antiapoptotic protein that preserves protein during cellular stress. In prostate cancer, clusterin overexpression is associated with a high Gleason score and has been detected in patients with mCRPC after neoadjuvant hormone therapy.

Studies of clusterin have demonstrated its antiapoptotic and prosurvival activities in prostate cancer that are believed to be associated with docetaxel resistance [74]. In a phase II trial custirsen (weekly intravenous administered) plus docetaxel extended median survival rates from 16.9 months to 23.8 months compared with single-agent docetaxel [75] [76], and a decrease of clusterin level after custirsen treatment was observed. In another Phase II trial custirsen was administered in combination with docetaxel or mitoxantrone as a second-line therapy in patients with mCRPC progressing after first-line docetaxel. Both combinations were well tolerated, but OS and PFS were better in the docetaxel arm (15.8 and 7.2 months vs 11.5 and 3.4 months, respectively) [76].

A randomized open-label phase III trial (SYNERGY) evaluated first-line therapy with custirsen in combination with docetaxel-prednisone versus docetaxel-prednisone alone in chemotherapy-naive mCRPC patients [77]. In particular, CRPC patients with a poor prognosis appeared to benefit from custirsen when added to docetaxel as 1st-line therapy. The poor prognosis group was analyzed separately for treatment effect $(n=492)$. The median OS was $17.0 \mathrm{~m}$ in the custirsen arm vs. $14.0 \mathrm{~m}$ in the control arm. PSA progression in the poor prognosis group also favored custirsen. Side effects included febrile neutropenia, fever, pleural effusion, and dyspnea [77].

Currently another randomized open-label phase III trial is ongoing in order to evaluate the OS, as first end point. In particular, AFFINITY trial, investigates the survival benefit in docetaxel-pretreated patients of second-line chemotherapy with cabazitaxel $25 \mathrm{mg} / \mathrm{m}^{2}$ and prednisone $10 \mathrm{mg} /$ day with or without custirsen [78].

\section{Conclusions}

The treatment of prostate cancer, in particular of its most malignant hormone independent and castration resistant forms, has been improved thanks to the develop of new strategies.

In the last few years, many different therapeutic strategies for CRPC have been developed and evaluated in clinical studies. Several strategies showed objective clinical benefit and have been approved for clinical use. These include the androgen inhibitors such as enzalutamide and abiraterone, radium-223 and sipuleucel- $\mathrm{T}$. Although enzalutamide, abiraterone and radium-223 represent the standard care for the treatment of bone metastatic CRPC, sipuleucel-T has not widely used in clinical practice because, rarely, patients responded to therapy. The identification of biomarkers could help to select patients that may (or may not) benefit from sipuleucel-T therapy.

In the near future, it will be crucial to test these agents together or sequentially. In this regard a Phase II study on CRPC bone metastatic patients in which abiraterone and enzalutamide were administered simultaneously showed promising results [73]. Moreover, recent evidence has demonstrated that anti-androgens are able to tar- 
get both prostate cancer cells and the bone microenvironment. This could influence future therapeutic approaches evaluating the possibility of combining anti-androgen treatment with bone-targeted agents (biphosphonates, denosumab) in order to achieve better control of prostate cancer bone metastases. In recent years, the simultaneous development of novel and more potent classes of drugs targeting androgen pathway has emerged such as ortonel, ARN-509 and galeterone.

On the other hand, there are several examples of new molecular targeting drugs that showed promising results in preclinical PCa models but showed insufficient effects in clinical phase III studies. These targets include the endothelin A receptor antagonist zibotentan, the Src inhibitor dasatinib, and the c-MET inhibitor cabozantinib, although some of the initial excitement failed to materialize.

In spite of the development and consequent approval of a number of new drugs active against mCRPC, no great improvement in overall was found and several patients have disease progression and early mortality. The discovery of new drug resistance mechanisms, the genomic and proteomic analysis to classify different prostate cancer molecular subtypes, as well as the set-up of new prognostic and predictive biomarkers, may further improve mCRPC treatment.

In order to make a great deal of progress in prostate cancer treatment, it is imperative that both basic and clinical investigators cooperate to reach this common goal.

\section{References}

[1] Arnold, M., Karim-Kos, H.E., Coebergh, J.W., et al. (2015) Recent Trends in Incidence of Five Common Cancers in 26 European Countries since 1988: Analysis of the European Cancer Observatory. European Journal of Cancer, 51, 1164-1187. http://dx.doi.org/10.1016/j.ejca.2013.09.002

[2] Siegel, R.L., Miller, K.D. and Jemal, A. (2015) Cancer Statistics, 2015. CA: A Cancer Journal for Clinicians, 65, 5-29. http://dx.doi.org/10.3322/caac.21254

[3] Bostwick, D.G. (1995) High-Grade Prostatic Intraepithelial Neoplasia: The Most Likely Precursor of Prostate Cancer. Cancer, 75, 1823-1836. http://dx.doi.org/10.1002/1097-0142(19950401)75:7+<1823::AID-CNCR2820751612>3.0.CO;2-7

[4] Heidenreich, A., Bellmunt, J., Bolla, M., et al. (2011) EAU Guidelines on Prostate Cancer. Part 1: Screening, Diagnosis, and Treatment of Clinically Localised Disease. European Urology, 59, 61-71. http://dx.doi.org/10.1016/j.eururo.2010.10.039

[5] Damber, J.E. (2005) Endocrine Therapy for Prostate Cancer. Acta Oncologica, 44, 605-609. http://dx.doi.org/10.1080/02841860510029743

[6] Keller, E.T., Zhang, J., Cooper, C.R., et al. (2001) Prostate Carcinoma Skeletal Metastases: Cross-Talk between Tumor and Bone. Cancer and Metastasis Reviews, 20, 333-349. http://dx.doi.org/10.1023/A:1015599831232

[7] Mundy, G.R. (2002) Metastasis to Bone: Causes, Consequences and Therapeutic Opportunities. Nature Reviews Cancer, 2, 584-593. http://dx.doi.org/10.1038/nrc867

[8] Kirby, M., Hirst, C. and Crawford, E.D. (2011) Characterising the Castration-Resistant Prostate Cancer Population: A Systematic Review. International Journal of Clinical Practice, 65, 1180-1192. http://dx.doi.org/10.1111/j.1742-1241.2011.02799.x

[9] de Bono, J.S., Oudard, S., Ozguroglu, M., et al. (2010) Prednisone plus Cabazitaxel or Mitoxantrone for Metastatic Castration-Resistant Prostate Cancer Progressing after Docetaxel Treatment: A Randomised Open-Label Trial. Lancet, 376, 1147-1154. http://dx.doi.org/10.1016/S0140-6736(10)61389-X

[10] de Bono, J.S., Logothetis, C.J., Molina, et al. (2011) Abiraterone and Increased Survival Inmetastatic Prostate Cancer. New England Journal of Medicine, 364, 1995-2005. http://dx.doi.org/10.1056/NEJMoa1014618

[11] Scher, H.I., Fizazi, K., Saad, F., et al. (2012) Increased Survival with Enzalutamide in Prostate Cancer after Chemotherapy. New England Journal of Medicine, 367, 1187-1197. http://dx.doi.org/10.1056/NEJMoa1207506

[12] Parker, C., Nilsson, S., Heinrich, D., et al. (2013) Alpha Emitter Radium-223 and Survival in Metastatic Prostate Cancer. New England Journal of Medicine, 369, 213-223. http://dx.doi.org/10.1056/NEJMoa1213755

[13] Fizazi, K., Carducci, M., Smith, M., et al. (2011) Denosumab versus Zoledronic Acid for Treatment of Bone Metastases in Men with Castration-Resistant Prostate Cancer: A Randomised, Double-Blind Study. Lancet, 377, 813-822. http://dx.doi.org/10.1016/S0140-6736(10)62344-6

[14] Kantoff, P.W., Higano, C.S., Shore, N.D., et al. (2010) Sipuleucel-T Immunotherapy for Castration Resistant Prostate Cancer. New England Journal of Medicine, 363, 411-422. http://dx.doi.org/10.1056/NEJMoa1001294

[15] Montgomery, R.B., Mostaghel, E.A., Vessella, R., et al. (2008) Maintenance of Intratumoral Androgens in Metastatic 
Prostate Cancer: A Mechanism for Castration-Resistant Tumor Growth. Cancer Research, 68, 4447-4454. http://dx.doi.org/10.1158/0008-5472.CAN-08-0249

[16] Coffey, K. and Robson, C.N. (2012) Regulation of the Androgen Receptor by Post-Translational Modifications. Journal of Endocrinology, 215, 221-237. http://dx.doi.org/10.1530/JOE-12-0238

[17] Liu, L.L., Xie, N., Sun, S., Plymate, S., Mostaghel, E. and Dong, X. (2014) Mechanisms of the Androgen Receptor Splicing in Prostate Cancer Cells. Oncogene, 33, 3140-3150. http://dx.doi.org/10.1038/onc.2013.284

[18] Waltering, K.K., Urbanucci, A. and Visakorpi, T. (2012) Androgen Receptor (AR) Aberrations in Castration-Resistant Prostate Cancer. Molecular and Cellular Endocrinology, 360, 38-43. http://dx.doi.org/10.1016/j.mce.2011.12.019

[19] Drake, J.M., Graham, N.A., Stoyanova, T., et al. (2012) Oncogene-Specific Activation of Tyrosine Kinase Networks during Prostate Cancer Progression. Proceedings of the National Academy of Sciences of the United States of America, 109, 1643-1648. http://dx.doi.org/10.1073/pnas.1120985109

[20] Rowlands, M.G., Barrie, S.E., Chan, F., et al. (1995) Esters of 3-Pyridylacetic Acid That Combine Potent Inhibition of 17.alpha.-Hydroxylase/C17,20-Lyase (Cytochrome P45017.alpha.) with Resistance to Esterase Hydrolysis. Journal of Medicinal Chemistry, 38, 4191-4197. http://dx.doi.org/10.1021/jm00021a008

[21] O’Donnell, A., Judson, I., Dowsett, M., et al. (2004) Hormonal Impact of the 17alpha-Hydroxylase/C(17,20)-Lyase Inhibitor Abiraterone Acetate (CB7630) in Patients with Prostate Cancer. British Journal of Cancer, 90, 2317-2325.

[22] Barrie, S.E., Potter, G.A., Goddard, P.M., Haynes, B.P., Dowsett, M. and Jarman, M. (1994) Pharmacology of Novel Steroidal Inhibitors of Cytochrome $\mathrm{P}_{50} \mathrm{H}_{17 \alpha}$ (17 $\alpha$-Hydroxylase/C17-20 Lyase). The Journal of Steroid Biochemistry and Molecular Biology, 50, 267-273. http://dx.doi.org/10.1016/0960-0760(94)90131-7

[23] Attard, G., Reid, A.H., Auchus, R.J., et al. (2012) Clinical and Biochemical Consequences of CYP17A1 Inhibition with Abiraterone Given with and without Exogenous Glucocorticoids in Castrate Men with Advanced Prostate Cancer. The Journal of Clinical Endocrinology \& Metabolism, 97, 507-516. http://dx.doi.org/10.1210/jc.2011-2189

[24] Ryan, C.J., Smith, M.R., de Bono, J.S., et al. (2013) Abiraterone in Metastatic Prostate Cancer without Previous Chemotherapy. The New England Journal of Medicine, 368, 138-148. http://dx.doi.org/10.1056/NEJMoa1209096

[25] Ryan, C.J., Smith, M.R., Fizazi, K., et al. (2015) Abiraterone Acetate plus Prednisone versus Placebo plus Prednisone in Chemotherapy-Naive Men with Metastatic Castration-Resistant Prostate Cancer (COU-AA-302): Final Overall Survival Analysis of a Randomized, Double-Blind, Placebo-Controlled Phase 3 Study. The Lancet Oncology, 16, 152-160. http://dx.doi.org/10.1016/S1470-2045(14)71205-7

[26] Iuliani, M., Pantano, F., Buttigliero, C., et al. (2015) Biological and Clinical Effects of Abiraterone on Anti-Resorptive and Anabolic Activity in Bone Microenvironment. Oncotarget, 6, 12520-12528. http://dx.doi.org/10.18632/oncotarget.3724

[27] Niraula, S., Chi, K. and Joshua, A.M. (2012) Beyond Castration-Defining Future Directions in the Hormonal Treatment of Prostate Cancer. Hormones and Cancer, 3, 3-13.

[28] Dreicer, R., Maclean, D., Suri, A., et al. (2014) Phase I/II Trial of Orteronel (TAK-700)—An Investigational 17,20Lyase Inhibitor-In Patients with Metastatic Castration-Resistant Prostate Cancer. Clinical Cancer Research, 20, 1335-1344.

[29] Dreicer, R., Jones, R., Oudard, S., et al. (2014) Results from a Phase 3, Randomized, Double-Blind, Multicenter, Placebo-Controlled Trial of Orteronel (TAK-700) plus Prednisone in Patients with Metastatic Castration-Resistant Prostate Cancer (mCRPC) That Has Progressed during or Following Docetaxel-Based Therapy (ELM-PC 5 trial). 2014 Genitourinary Cancers Symposium, San Francisco, 29 January-2 February 2014.

[30] Taplin, M., Chu, F., Morrison, J., Pili, R., Rettig, M., Stephenson, J., Vogelzang, N. and Montgomery, R. (2012) Abstract CT-07: ARMOR1: safety of Galeterone (TOK-001) in a Phase 1 Clinical Trial in Chemotherapy Naïve Patients with Castration Resistant Prostate Cancer (CRPC). Cancer Research, 72, CT-07.

[31] Taplin, M.E., Montgomery, R.B. and ARMOR2 Group (2014) ARMOR2: Galeterone in Progressive CRPC Patients Who Have Failed Oral Therapy. 2014 Genitourinary Cancers Symposium, San Francisco, 29 January-2 February 2014.

[32] Clegg, N.J., Wongvipat, J., Joseph, J.D., et al. (2012) ARN-509: A Novel Antiandrogen for Prostate Cancer Treatment. Cancer Research, 72, 1494-1503. http://dx.doi.org/10.1158/0008-5472.CAN-11-3948

[33] Rathkopf, D.E., Morris, M.J., Fox, J.J., et al. (2013) Phase I Study of ARN-509, a Novel Antiandrogen, in the Treatment of Castration-Resistant Prostate Cancer. Journal of Clinical Oncology, 31, 3525-3530.

[34] Leibowitz-Amit, R. and Joshua, A.M. (2012) Targeting the Androgen Receptor in the Management of Castration-Resistant Prostate Cancer: Rationale, Progress, and Future Directions. Current Oncology, 19, 22-31.

[35] Garcia, J.A., Kataja, V.V., James, N.D., et al. (2014) Bone and Soft Tissue Response from a Phase I/II Study with ODM-201 in Metastatic Castration-Resistant Prostate Cancer (mCRPC). 2014 Genitourinary Cancer Symposium, San Francisco, 29 January-2 February 2014. 
[36] Dai, J.L., Hall, C.L., Escara-Wilke, J., Mizokami, A., Keller, J.M. and Keller, E.T. (2008) Prostate Cancer Induces Bone Metastasis through Wnt-Induced Bone Morphogenetic Protein-Dependent and Independent Mechanisms. Cancer Research, 68, 5785-5794. http://dx.doi.org/10.1158/0008-5472.CAN-07-6541

[37] Hall, C.L., Kang, S., MacDougald, O.A. and Keller, E.T. (2006) Role of Wnts in Prostate Cancer Bone Metastases. Journal of Cellular Biochemistry, 97, 661-672.

[38] Kingsley, L.A., Fournier, P.G., Chirgwin, J.M. and Guise, T.A. (2007) Molecular Biology of Bone Metastasis. Molecular Cancer Therapeutics, 6, 2609-2617.

[39] Tucci, M., Mosca, A., Lamanna, G., et al. (2009) Prognostic Significance of Disordered Calcium Metabolism in Hormone-Refractory Prostate Cancer Patients with Metastatic Bone Disease. Prostate Cancer and Prostatic Diseases, 12, 94-99.

[40] Coleman, R., Gnant, M., Morgan, G. and Clezardin, P. (2012) Effects of Bone-Targeted Agents on Cancer Progression and Mortality. Journal of the National Cancer Institute, 104, 1059-1067. http://dx.doi.org/10.1093/jnci/djs263

[41] Coleman, R.E. and McCloskey, E.V. (2011) Bisphosphonates in Oncology. Bone, 49, 71-76.

[42] Saad, F., Gleason, D.M., Murray, R., Tchekmedyian, S., Venner, P., Lacombe, L., Chin, J.L., Vinholes, J.J., Goas, J.A., Zheng, M. and For the Zoledronic Acid Prostate Cancer Study Group (2004) Long-Term Efficacy of Zoledronic Acid for the Prevention of Skeletal Complications in Patients with Metastatic Hormone-Refractory Prostate Cancer. Journal of the National Cancer Institute, 96, 879-882. http://dx.doi.org/10.1093/jnci/djh141

[43] Guise, T.A. (2000) Molecular Mechanisms of Osteolytic Bone Metastases. Cancer, 88, 2892-2898.

[44] Mori, K., Le Goff, B., Charrier, C., Battaglia, S., Heymann, D. and Rédini, F. (2007) DU145 Human Prostate Cancer Cells Express Functional Receptor Activator of NFאB: New Insights in the Prostate Cancer Bone Metastasis Process. Bone, 40, 981-990. http://dx.doi.org/10.1016/j.bone.2006.11.006

[45] Lipton, A., Fizazi, K., Stopeck, A.T., et al. (2012) Superiority of Denosumab to Zoledronic Acid for Prevention of Skeletal-Related Events: A Combined Analysis of 3 Pivotal, Randomised, Phase 3 Trials. European Journal of Cancer, 48, 3082-3092. http://dx.doi.org/10.1016/j.ejca.2012.08.002

[46] Silberstein, E.B. (1996) Dosage and Response in Radiopharmaceutical Therapy of Painful Osseous Metastases. Journal of Nuclear Medicine, 37, 249-252.

[47] James, N.D., Pirrie, S., Barton, D., et al. (2013) Clinical Outcomes in Patients with Castrate-Refractory Prostate Cancer (CRPC) Metastatic to Bone Randomized in the Factorial TRAPEZE Trial to Docetaxel (D) with Strontium-89 (Sr89), Zoledronic Acid (ZA), Neither, or Both (ISRCTN 12808747). Journal of Clinical Oncology, 31.

[48] Allen, B.J. (2008) Clinical Trials of Targeted Alpha Therapy for Cancer. Reviews on Recent Clinical Trials, 3, $185-191$. http://dx.doi.org/10.2174/157488708785700339

[49] Henriksen, G., Breistøl, K., Bruland, Ø.S., Fodstad, Ø. and Larsen, R.H. (2002) Significant Antitumor Effect from Bone-Seeking, Alpha-Particle-Emitting (223)Ra Demonstrated in an Experimental Skeletal Metastases Model. Cancer Research, 62, 3120-3125.

[50] Nilsson, S., Franzén, L., Parker, C., et al. (2007) Bone-Targeted Radium-223 in Symptomatic, Hormone-Refractory Prostate Cancer: A Randomised, Multicentre, Placebo-Controlled Phase II Study. The Lancet Oncology, 8, 587-594. http://dx.doi.org/10.1016/S1470-2045(07)70147-X

[51] Larsen, R.H., Saxtorph, H., Skydsgaard, M., et al. (2006) Radiotoxicity of the Alpha-Emitting Bone-Seeker 223Ra Injected Intravenously into Mice: Histology, Clinical Chemistry and Hematology. In Vivo, 20, 325-331.

[52] Zhang, S., Zhau, H.E., Osunkoya, A.O., et al. (2010) Vascular Endothelial Growth Factor Regulates Myeloid Cell Leukemia-1 Expression through Neuropilin-1-Dependent Activation of c-MET Signaling in Human Prostate Cancer Cells. Molecular Cancer, 9, 9. http://dx.doi.org/10.1186/1476-4598-9-9

[53] Knudsen, B.S., Gmyrek, G.A., Inra, J., et al. (2002) High Expression of the Met Receptor in Prostate Cancer Metastasis to Bone. Urology, 60, 1113-1117.

[54] Smith, M.R., De Bono, J.S., Sternberg, C.N., et al. (2015) Final Analysis of COMET-1: Cabozantinib (Cabo) versus Prednisone (Pred) in Metastatic Castration-Resistant Prostate Cancer (mCRPC) Patients (pts) Previously Treated with Docetaxel (D) and Abiraterone (A) and/or Enzalutamide (E). 2015 Genitourinary Cancers Symposium.

[55] Tatarov, O., Mitchell, T.J., Seywright, M., Leung, H.Y., Brunton, V.G. and Edwards, J. (2009) SRC Family Kinase Activity Is Up-Regulated in Hormone-Refractory Prostate Cancer. Clinical Cancer Research, 15, 3540-3549. http://dx.doi.org/10.1158/1078-0432.CCR-08-1857

[56] Zaidi, S.K., Sullivan, A.J., Medina, R., et al. (2004) Tyrosine Phosphorylation Controls Runx2-Mediated Subnuclear Targeting of YAP to Repress Transcription. The EMBO Journal, 23, 790-799. http://dx.doi.org/10.1038/sj.emboj.7600073

[57] Koreckij, T., Nguyen, H., Brown, L.G., Yu, E.Y., Vessella, R.L. and Corey, E. (2009) Dasatinib Inhibits the Growth of 
Prostate Cancer in Bone and Provides Additional Protection from Osteolysis. British Journal of Cancer, 101, $263-268$. http://dx.doi.org/10.1038/sj.bjc.6605178

[58] Nelson, J.B., Love, W., Chin, J.L., et al. (2008) Phase 3, Randomized, Controlled Trial of Atrasentan in Patients with Nonmetastatic, Hormone-Refractory Prostate Cancer. Cancer, 113, 2478-2487. http://dx.doi.org/10.1002/cncr.23864

[59] Araujo, J.C., Trudel, G.C., Saad, F., et al. (2013) Docetaxel and Dasatinib or Placebo in Men with Metastatic Castration-Resistant Prostate Cancer (READY): A Randomised, Double-Blind Phase 3 Trial. The Lancet Oncology, 14, 1307-1316. http://dx.doi.org/10.1016/S1470-2045(13)70479-0

[60] Nelson, J., Bagnato, A., Battistini, B. and Nisen, P. (2003) The Endothelin Axis: Emerging Role in Cancer. Nature Reviews Cancer, 3, 110-116. http://dx.doi.org/10.1038/nrc990

[61] Logothetis, C.J. and Lin, S.H. (2005) Osteoblasts in Prostate Cancer Metastasis to Bone. Nature Reviews Cancer, 5, 21-28. http://dx.doi.org/10.1038/nrc1528

[62] Nelson, J.B., Fizazi, K., Miller, K., et al. (2012) Phase 3, Randomized, Placebo-controlled Study of Zibotentan (ZD4054) in Patients with Castration-Resistant Prostate Cancer Metastatic to Bone. Cancer, 118, 5709-5718. http://dx.doi.org/10.1002/cncr.27674

[63] Fizazi, K.S., Higano, C.S., Nelson, J.B., et al. (2013) Phase III, Randomized, Placebo-Controlled Study of Docetaxel in Combination with Zibotentan in Patients with Metastatic Castration-Resistant Prostate Cancer. Journal of Clinical Oncology, 31, 1740-1747. http://dx.doi.org/10.1200/JCO.2012.46.4149

[64] Taylor, B.S., Varambally, S. and Chinnaiyan, A.M. (2006) Differential Proteomic Alterations between Localised and Metastatic Prostate Cancer. British Journal of Cancer, 95, 425-430. http://dx.doi.org/10.1038/sj.bjc.6603274

[65] Pardoll, D.M. (2012) The Blockade of Immune Checkpoints in Cancer Immunotherapy. Nature Reviews Cancer, 12, 252-264. http://dx.doi.org/10.1038/nrc3239

[66] Tollefson, M.K., Karnes, R.J., Thompson, R.H., et al. (2010) A Randomized Phase II Study of Ipilimumab with Androgen Ablation Compared with Androgen Ablation Alone in Patients with Advanced Prostate Cancer. 2010 Genitourinary Cancers Symposium, San Francisco, 5-7 March 2010.

[67] Kwon, E.D., Drake, C.G., Scher, H.I., et al. (2014) Ipilimumab versus Placebo after Radiotherapy in Patients with Metastatic Castration-Resistant Prostate Cancer That Had Progressed after Docetaxel Chemotherapy (CA184-043): A Multicentre, Randomised, Double-Blind, Phase 3 Trial. The Lancet Oncology, 15, 700-712. http://dx.doi.org/10.1016/S1470-2045(14)70189-5

[68] Higano, C.S., Schellhammer, P.F., Small, E.J., et al. (2009) Integrated Data from 2 Randomized, Double-Blind, Placebo-Controlled, Phase 3 Trials of Active Cellular Immunotherapy with Sipuleucel-T in Advanced Prostate Cancer. Cancer, 115, 3670-3679. http://dx.doi.org/10.1002/cncr.24429

[69] Isaacs, J.T. (2010) The Long and Winding Road for the Development of Tasquinimod as an Oral Second-Generation Quinoline-3-Carboxamide Antiangiogenic Drug for the Treatment of Prostate Cancer. Expert Opinion on Investigational Drugs, 19, 1235-1243. http://dx.doi.org/10.1517/13543784.2010.514262

[70] Raymond, E., Dalgleish, A., Damber, J.-E., Smith, M. and Pili, R. (2014) Mechanisms of Action of Tasquinimod on the Tumour Microenvironment. Cancer Chemotherapy and Pharmacology, 73, 1-8. http://dx.doi.org/10.1007/s00280-013-2321-8

[71] Pili, R., Haggman, M., Stadler, W.M., et al. (2011) Phase II Randomized, Double-Blind, Placebo Controlled Study of Tasquinimod in Men with Minimally Symptomatic Metastatic Castrate-Resistant Prostate Cancer. Journal of Clinical Oncology, 29, 4022-4028. http://dx.doi.org/10.1200/JCO.2011.35.6295

[72] Clinical Trial Number NCT01234311 for “A Study of Tasquinimod in Men with Metastatic Castrate Resistant Prostate Cancer" at ClinicalTrials.gov

[73] Zielinski, R. and Chi, K.N. (2012) Custirsen (OGX-011): A Second-Generation Antisense Inhibitor of Clusterin in Development for the Treatment of Prostate Cancer. Future Oncology, 8, 1239-1251. http://dx.doi.org/10.2217/fon.12.129

[74] Higano, C.S. (2013) Potential Use of Custirsen to Treat Prostate Cancer. OncoTargets and Therapy, 6, $785-797$. http://dx.doi.org/10.2147/OTT.S33077

[75] Chi, K.N., Bjartell, A., Dearnaley, D., et al. (2009) Castration-Resistant Prostate Cancer: From New Pathophysiology to New Treatment Targets. European Urology, 56, 594-605. http://dx.doi.org/10.1016/j.eururo.2009.06.027

[76] Chi, K.N., Hotte, S.J., Yu, E.Y., et al. (2010) Randomized Phase II Study of Docetaxel and Prednisone with or without OGX-011 in Patients With Metastatic Castration-Resistant Prostate Cancer. Journal of Clinical Oncology, 28, 42474254. http://dx.doi.org/10.1200/JCO.2009.26.8771

[77] Chi, K.N., Higano, C.S., Blumenstein, B.A., et al. (2015) Phase III SYNERGY Trial: Docetaxel +/- Custirsen and Overall Survival in Patients (pts) with Metastatic Castration-Resistant Prostate Cancer (mCRPC) and Poor Prognosis. 
2015 ASCO Annual Meeting.

[78] Clinical Trial Number NCT01578655 for “A Study of Tasquinimod in Men with Metastatic Castrate Resistant Prostate Cancer” at ClinicalTrials.gov.

\section{Abbreviations}

PCa: Prostate Cancer

PIN: Prostatic Intraepithelial Neoplasia

HGPIN: High Grade Prostatic Intraepithelial Neoplasia

ADT: Androgen Deprivation Therapies

PSA: Prostate Specific Antigen

SRE: Skeletal Related Event

mCRPC: metastatic Castration Resistant Prostate Cancer

OS: Overall Survival

AR: Androgen Receptor

CYP17: Cytocrome P450 17 alpha hydroxysteroid dehydrogenase

PFS: Progression Free Survival

RANK: Receptor Activator of Nuclear Factor-Kb

RANKL: Receptor Activator of Nuclear Factor-kB Ligand

OPG: Osteoprotegerin

TNF: Tumor Necrosis Factor

TGF-b: Tumor Growth Factor-b Factor

PDGF: Platelet-Derived Growth Factor

bALP: bone alkaline phosphatase

uNTX: urine N-telopeptide

HGF: Hepatocyte Growth Factor

VEGF: Vascular Endothelial Growth Factor

VEGFR: Vascular Endothelial Growth Factor Receptor

ET: Endothelin

TAAs: Tumor Associated Antigens

CTLA-4: Cytotoxic T-lymphocyte associated antigen 4

PAP-GM-CSF: Phosphatase-granulocyte macrophage colony-stimulating factor

HIF-1 $\alpha$ : Hypoxia-Inducible Factor 1-alpha 